\title{
Analysis of genetic variants of dyslexia candidate genes KIAAO319 and DCDC2 in Indian population
}

\author{
Shyamala K Venkatesh ${ }^{1}$, Anand Siddaiah ${ }^{2}$, Prakash Padakannaya ${ }^{2}$ and Nallur B Ramachandra ${ }^{1}$ \\ Developmental dyslexia (DD) is a heritable, complex genetic disorder associated with impairment in reading and writing \\ skills despite having normal intellectual ability and appropriate educational opportunities. Chromosome 6p23-21.3 at DYX2 \\ locus has showed the most consistent evidence of linkage for DD and two susceptible genes KIAAO319 and DCDC2 for DD at \\ DYX2 locus showed significant association. Specific candidate gene-association studies have identified variants, risk haplotypes \\ and microsatellites of KIAAO319 and $D C D C 2$ correlated with wide range of reading-related traits. In this study, we used a \\ case-control approach for analyzing single-nucleotide polymorphisms (SNPs) in KIAAO319 and DCDC2. Our study \\ demonstrated the association of DD with SNP rs4504469 of KIAA0319 and not with any SNPs of DCDC2. \\ Journal of Human Genetics (2013) 58, 531-538; doi:10.1038/jhg.2013.46; published online 16 May 2013
}

Keywords: case-control; developmental dyslexia; KIAA0319 and DCDC2; MassARRAY; SNPS

\section{INTRODUCTION}

Developmental dyslexia (DD) is a heritable, complex genetic disorder that affects $5-12 \%$ of school age children. ${ }^{1}$ The corresponding figure for South India, where the current study was carried out is reported to be $9.87 \% .^{2}$ Individuals with dyslexia show impairment in reading and writing skills despite having normal intellectual ability and appropriate educational opportunities. ${ }^{3}$ Linkage studies have identified nine loci and several genes, located in different chromosomal regions that are associated with DD. ${ }^{4}$ More than ten genes that contribute to the disorder are involved in neuronal migration. ${ }^{5}$ Chromosome 6p23-21.3 at DYX2 locus has showed the most consistent evidence of linkage for DD. ${ }^{6-10}$ Specific candidate gene-association studies have identified variants, risk haplotypes and microsatellites of KIAA0319 and DCDC2 correlated with wide range of reading-related traits. ${ }^{11-19}$

Dyslexia-KIAA0319 association was first identified in Colorado Learning Disabilities Research Center (CLDRC) samples by identifying microsatellite marker residing in KIAA0319. ${ }^{9}$ Later, examination of the 77-kb region of KIAA0319 and TTRAP found significant association of a few single-nucleotide polymorphisms (SNPs) with various reading-related measures. ${ }^{11}$ SNP rs4504469 was the only coding nonsynonymous mutation found associated significantly with phonological decoding, single-word reading and phonological awareness skills. ${ }^{11}$ A specific three-marker haplotype, rs4504469/ rs2038137/rs2143340 was significantly associated with irregular word reading. ${ }^{11}$ Further SNP genotyping was carried out in Cardiff and UK populations. ${ }^{12,20}$ Cardiff sample yielded significant evidence for the association of the SNP rs4504469 with DD and UK samples showed the strongest association for SNP rs761100 located in intronic region of KIAA0319. ${ }^{12,20}$ Another SNP rs3212236 located near the $5^{\prime}$-flanking region showed the most significant association with orthographic coding-irregular, reading and spelling. ${ }^{12,20}$ Functional analysis of SNPs (rs9461045, rs3212236, rs9467247) as well as risk haplotype rs4504469/rs2038137/rs2143340 demonstrated lowering expression of the KIAA0319. ${ }^{21,22}$ A study on markers across the $6 \mathrm{p}$ region also showed association with the markers in KIAA0319.23 A recent study of putative functional polymorphism analysis in Canadian subjects found support for previous studies indicating the 5' region of the KIAA0319 as the location of risk alleles contributing to DD. ${ }^{17}$

Screening of the SNPs in 440 UK families unselected for DD showed significant association of KIAA0319 SNP rs6935076 with poor reading performance in univariate analysis and rs2143340 of TTRAP showed significant association with both univariate and bivariate analysis. ${ }^{24}$ Haplotype analysis showed significant association with a three-SNP marker rs4504469/rs2038137/rs2143340. ${ }^{24}$ Replication of Francks et al. ${ }^{11}$ in general population showed associations of rs2143340 with poor reading, spelling, phonological awareness and non-word reading. ${ }^{25}$ These studies provided evidences for the influences of these genes on reading ability in the general population.

Dyslexia-DCDC2 association evidences were also obtained by the analysis of CLDRC samples. ${ }^{26}$ A large number of SNPs, haplotypes and deletions were identified in the intron of $D C D C 2$, which involved tandem repeats of putative brain-related transcription factor-binding sites significantly associated with multiple reading traits. ${ }^{10}$ Linkage disequilibrium study in German population also yielded significant association for the single and haplotype markers located in DCDC2 region. ${ }^{13}$ Further, analysis of DCDC2 SNPs and a 2445-bp deletion in

${ }^{1}$ Genetics and Genomics Laboratory, Department of Studies in Zoology, University of Mysore, Mysore, India and ${ }^{2}$ Department of Studies in Psychology, University of Mysore, Mysore, India

Correspondence: Dr NB Ramachandra, Genetics and Genomics Laboratory, Department of Studies in Zoology, University of Mysore, Manasagangotri, Mysore 570006, Karnataka, India. 

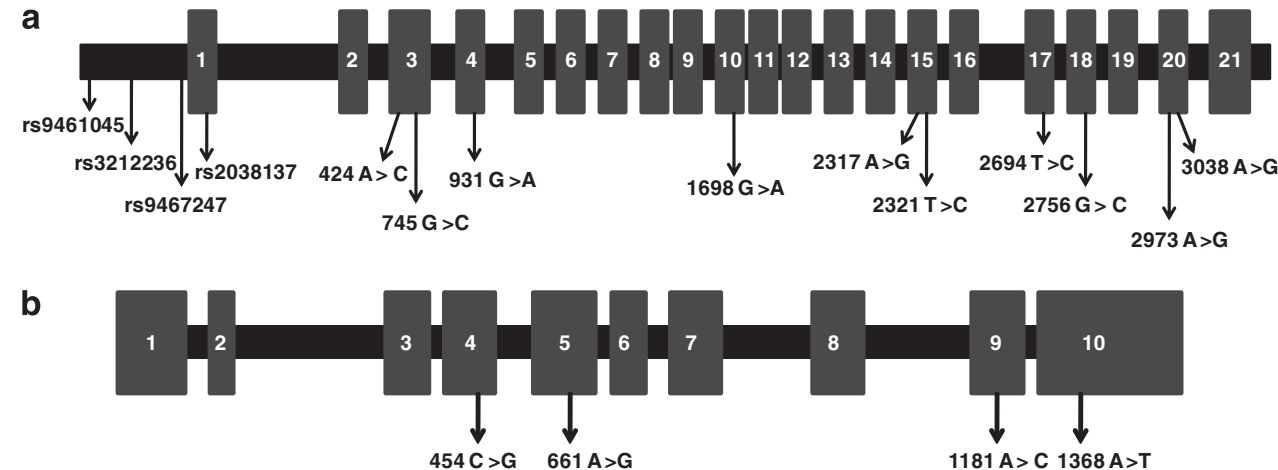

Figure 1 Gene structure and location of single-nucleotide polymorphisms (SNPs) screened in KIAAO319 and DCDC2. (a) Three SNPs of KIAAO319 located in the promoter region- rs9461045, rs3212236, rs9467247 and one SNP in $5^{\prime}$ UTR region rs2038137. The remaining 10 SNPs located in the exonic region, $424 \mathrm{~A}>\mathrm{C}, 745 \mathrm{G}>\mathrm{C}, 931 \mathrm{G}>\mathrm{A}, 1698 \mathrm{G}>\mathrm{A}, 2317 \mathrm{~A}>\mathrm{G}, 2321 \mathrm{~T}>\mathrm{C}, 2694 \mathrm{~T}>\mathrm{C}, 2756 \mathrm{G}>\mathrm{C}, 2973 \mathrm{~A}>\mathrm{G}, 3038 \mathrm{~A}>\mathrm{G}$. (b). All the four SNPs of $D C D C 2-$ rs33914824, rs2274305, rs34647318 and rs9460973 are located in the exonic region.

German population revealed strong association for three SNPs and deletion..$^{15}$ One intronic SNP of DCDC2 and three variants between DCDC2 and KIAA0319 were found significantly associated with the late mismatch negativity in 200 German children with dyslexia. ${ }^{27} \mathrm{~A}$ more recent study reported a significant association between BV677278 deletion with reading skills and memory. ${ }^{28}$ Finally, interaction between the markers of DCDC2 and KIAA0319 was identified in two independent samples of UK and Germany, ${ }^{20,29}$ though the association studies in the United States and China failed to replicate the previous studies of DCDC2 and KIAA0319. ${ }^{30,31} \mathrm{~A}$ recent meta-analysis study including four case-control studies and five TDT studies of SNPs of DCDC2 demonstrated that SNP rs807701 of $D C D C 2$ contribute significantly to the risk of dyslexia and no other DCDC2 polymorphisms were observed to increase the risk of dyslexia. $^{32}$

A recent study on epidemiological samples of Australia showed significant association for the SNP rs1419228 with regular word reading and spelling as well as irregular word reading, while SNP rs1091047 was found associated with irregular word reading. ${ }^{16}$ Four additional SNPs, namely rs9467075, rs9467076, rs7765678 and rs6922023, were found nominally associated with reading and spelling. This study identified $D C D C 2$ as a risk factor acts on normally varying reading skill in the epidemiological samples. ${ }^{16}$ Another study failed to identify the effects of KIAA0319 and $D C D C 2$ by analyzing previously reported markers in an epidemiological sample of 520 Australian individuals. ${ }^{33}$

In our previous study, screening of 14 SNPs of KIAA0319 and $D C D C 2$ in 50 cases and 50 controls showed a nonsignificant association. ${ }^{34}$ In this study, we have included the results of both previous and present study to explain the frequency of the SNPs in more number of samples. Our preliminary study was conducted with 10 SNPs of KIAA0319 and 4 SNPs of DCDC2 in 50 children with DD and 50 normal readers. The present study is an extended study wherein, we have screened additional four new SNPs of KIAA0319 in addition to the previously studied four SNPs in 160 children with dyslexia and 206 normal readers.

\section{MATERIALS AND METHODS}

\section{Subjects}

The study involved 210 ( 160 males and 50 females) children with DD certified by All India Institute of Speech and Hearing, Mysore and National Institute of Mental Health And Neurosciences, Bangalore. However, we tested these children on standard tests of reading and reading-related tests such as phonological awareness and rapid automatized naming (RAN) measures. All the children with dyslexia were normal on nonverbal intelligence measure and were reading at least two s.d. below the mean of reference group consisting of typically developing children of same age group. The performance of children with dyslexia on phonological awareness and RAN measures were also significantly poorer (individual scores differing more than two s.d. from the mean of reference group). In addition, they were also tested on measures of short-term memory, in which the children with dyslexia performed at two s.d. below the norm. Further, they all had normal or corrected to normal vision, and did not have any history of neurological disorders. The subjects were aged between 6 and 18 years with a mean age $15.52 \pm 3$. Among 210 cases, 33 were below 12 years and 177 were above 12 years. Age, gender ( 184 males and 72 females) and ethnicity matched 256 normal readers were also recruited for the study. Among 256 controls, 40 were below 12 years and 216 were above 12 years. Chi-square analysis between cases and controls for age and gender showed no significant $P$-value. Written informed consent forms were obtained from all the participating subjects/parents. Ethical approval was obtained from the ethical committee of University of Mysore. All the neuropsychological tests were administered individually in a quiet room either at school, at home or at the Psychology Department, University of Mysore in several sessions, lasting from $1-2 \mathrm{~h}$ each. Rapport was built and case history was collected in the first session. Later, nonverbal intelligence was assessed using standard progressive matrices. ${ }^{35}$ In the second session, reading measures such as Joshi and Aaron list ${ }^{36}$ and non-word-reading tests (prepared and used in a larger project; testretest reliability value $=0.974$, Cronbach's alpha $=0.781$ ), were administered along with Edinburgh handedness test. ${ }^{37}$ Word reading and spelling test, developed by Joshi and Aaron ${ }^{36}$ has 56 words belonging to different categories. There are 14 words each under regular, exception (irregular), unique and Morphophonemic word groups. RAN tests, phonological awareness tests and short-term memory tasks such as sentence repetition test from Cognitive Assessment Scale ${ }^{38}$ and Digit span test from PGIMS ${ }^{39}$ were administered in the third session. These were all psychometrically validated tests. These tests have been peer reviewed for content validity and have been used in other published studies by us. Total time taken to complete the tasks on reading measures and RAN tests were recorded along with the accuracy, whereas only accuracy scores were recorded for phonological awareness tasks.

\section{DNA extraction and genotyping}

Genomic DNA was isolated from peripheral blood leukocytes by using the Promega Wizard genomic DNA purification kit. The quality of DNA was ensured by spectrophotometry and agarose gel electrophoresis, visualized by ethidium bromide staining in gel documentation system. Genotyping of SNPs was carried out by MassARRAY with the help of Vimta Labs (Vimta Labs Ltd., Hyderabad, India) and Xcelris Labs (Xcelris Labs Ltd., Ahmedabad, India). A total of 18 SNPs from KIAA0319 and DCDC2 were selected based on their position in the gene $5^{\prime}$-UTR, exon, $3^{\prime}$-UTR and previous such association 
Table 1 Mean, s.d., Kurtosis, maximum and minimum scores on neuropsychological measure of children with developmental dyslexia and normal readers

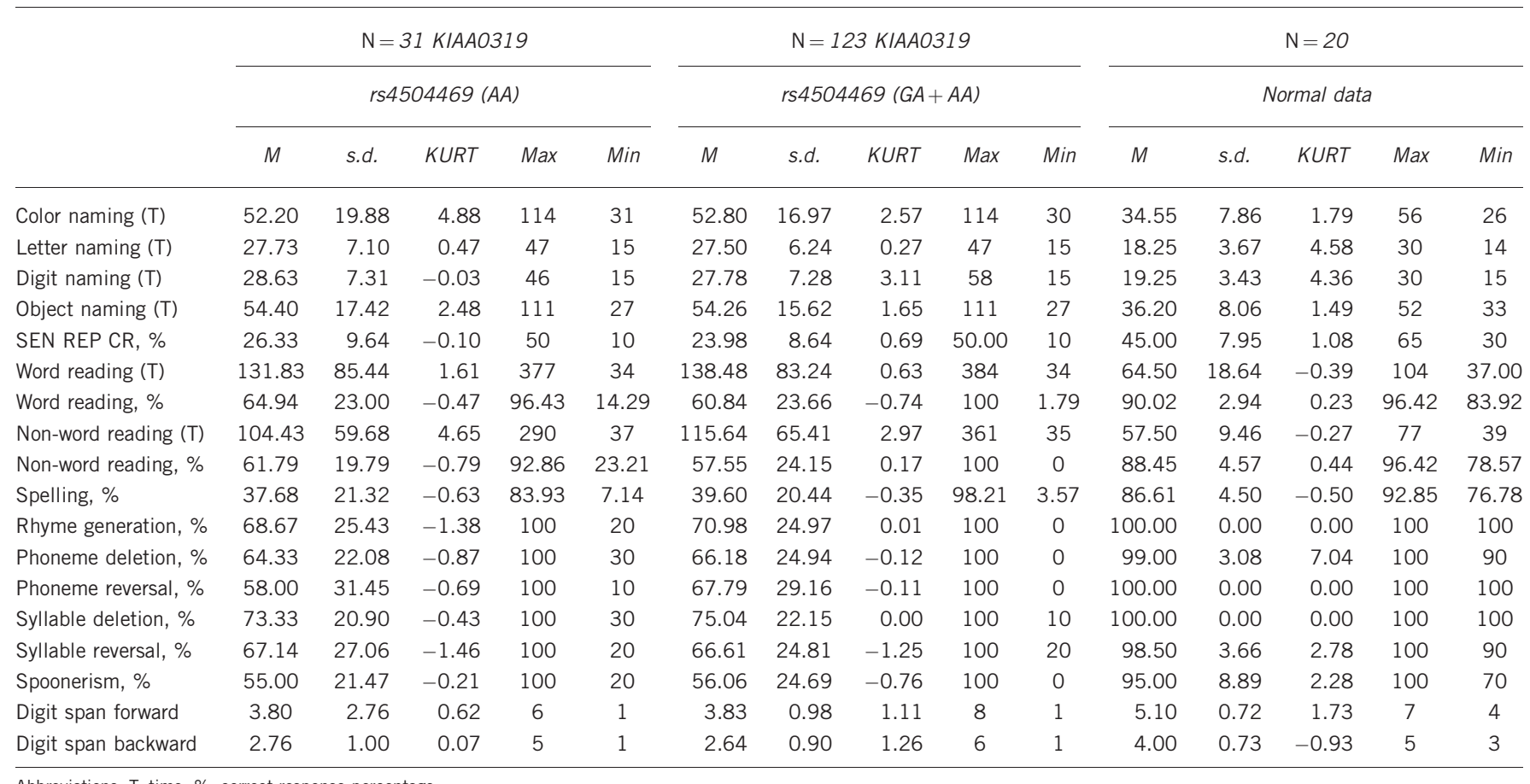

Abbreviations: T, time, \%, correct response percentage.

studies. We genotyped 11 SNPs reported by Francks et al., ${ }^{11} 1$ SNP from Dennis et al. ${ }^{22} 1$ SNP from Harold et al.,$^{20}$ and 1 SNP from NCBI database (Figure 1a). All the four coding SNPs of DCDC2 reported by Schumacher et al. ${ }^{13}$ were also selected (Figure 1b). SNPs rs2744559, rs2744550, rs2817191, rs10946705 and rs807535 of KIAA0319 and rs34647318 of DCDC2 were initially genotyped in 50 children with DD and 50 normal readers. Since, all individuals genotyped were homozygous for the normal alleles; these SNPs were not screened in the remaining samples. Initially genotyped SNP rs807541 of KIAA0319 was also not screened in the present study.

All SNPs were genotyped by MassARRAY Sequenom-iPLEXR Gold SNP genotyping platform with Spectro CHIPR and MALDI-TOF Mass spectrometer. Sequenom genotyping, PCR primers and primer extension probes were designed with the Spectro-DESIGNER software (Supplementary Table 1). Genotyping was performed in a 384-well sample plate using the homogeneous mass-extend assay facilitates simultaneous multiplex genotyping in a single experiment. PCR was performed on $10 \mathrm{ng}$ of DNA. Genotype data were obtained using SpectroTYPER software 4.0 (Sequenom).

\section{Statistical analysis}

Hardy-Weinberg equilibrium was tested for cases and controls separately by goodness of fit Chi-square test. Association analysis of frequencies of each allele and genotype with DD was done by Chi-square test, odds ratio (ORs) and $95 \%$ confidence intervals (CIs). The three specific genetic models (dominant, recessive and additive) were applied to SNPs to assess the association with risk of $\mathrm{DD}^{40}$ Bonferroni's correction was applied considering multiple testing for 18 SNPs and the correction was not applied if there was no significant chi-square $P$-value.

\section{RESULTS}

All the markers showed Hardy-Weinberg equilibrium $P$-value $>0.01$, except rs807534. Table 1 presents mean, s.d. and kurtosis along with maximum and minimum scores on various neuropsychological measures for 20 normal readers and children with DD in which SNP rs4504469 (genotype AA and GA + AA) was found. The mean differences between individuals with and without dyslexia on all the neuropsychological measures were found to be statistically significant.

\section{KIAA0319}

We identified a strong association for minor allele (A) frequency of rs4504469 with $P=0.009$ after applying Bonferroni's correction (Table 2). Significant association was also found for the SNP rs4504469 having homozygous genotype (AA) (odds ratio 2.53, 95\% confidence interval 1.36-4.71) with $P=0.036$ (after Bonferroni's correction) by comparing homozygous minor alleles of the SNP with the wild-type alleles (Table 3). Comparison of the heterozygous genotype of the SNP with wild-type SNP failed to show significant association after Bonferroni correction. Further association analyses of dominant, recessive and additive models showed association of same SNP rs4504469 in dominant model $(P=0.036)$ and additive model showed a trend toward significance $(P=0.054)$. Genotype GA/ AA frequency was found more in cases than the controls, whereas the wild-type genotype GG was found in more controls rather than cases (Table 4). The results of neuropsychological/cognitive tests showed poor performance on reading and spelling measures, RAN speed, phonemic segmentation tasks and short-term memory for both the homozygous genotype AA and GA + AA genotype of rs4504469. All the comparisons on neuropsychological/cognitive tests were made with reference to the mean performance of the control group (Figure 2).

SNP rs3212236 of KIAA0319 showed significant association in comparison of individual allele frequency, homozygous genotype analysis and recessive genotype model. However, this SNP did not show any significance in all the three analysis after applying Bonferroni's correction. SNP rs9467247 showed association in recessive and additive models but this SNP also failed to show any association after Bonferroni's correction. SNPs in rs4576240, 
Table 2 Allelic distributions of SNPs of KIAA0319 and DCDC2 among children with developmental dyslexia and normal readers

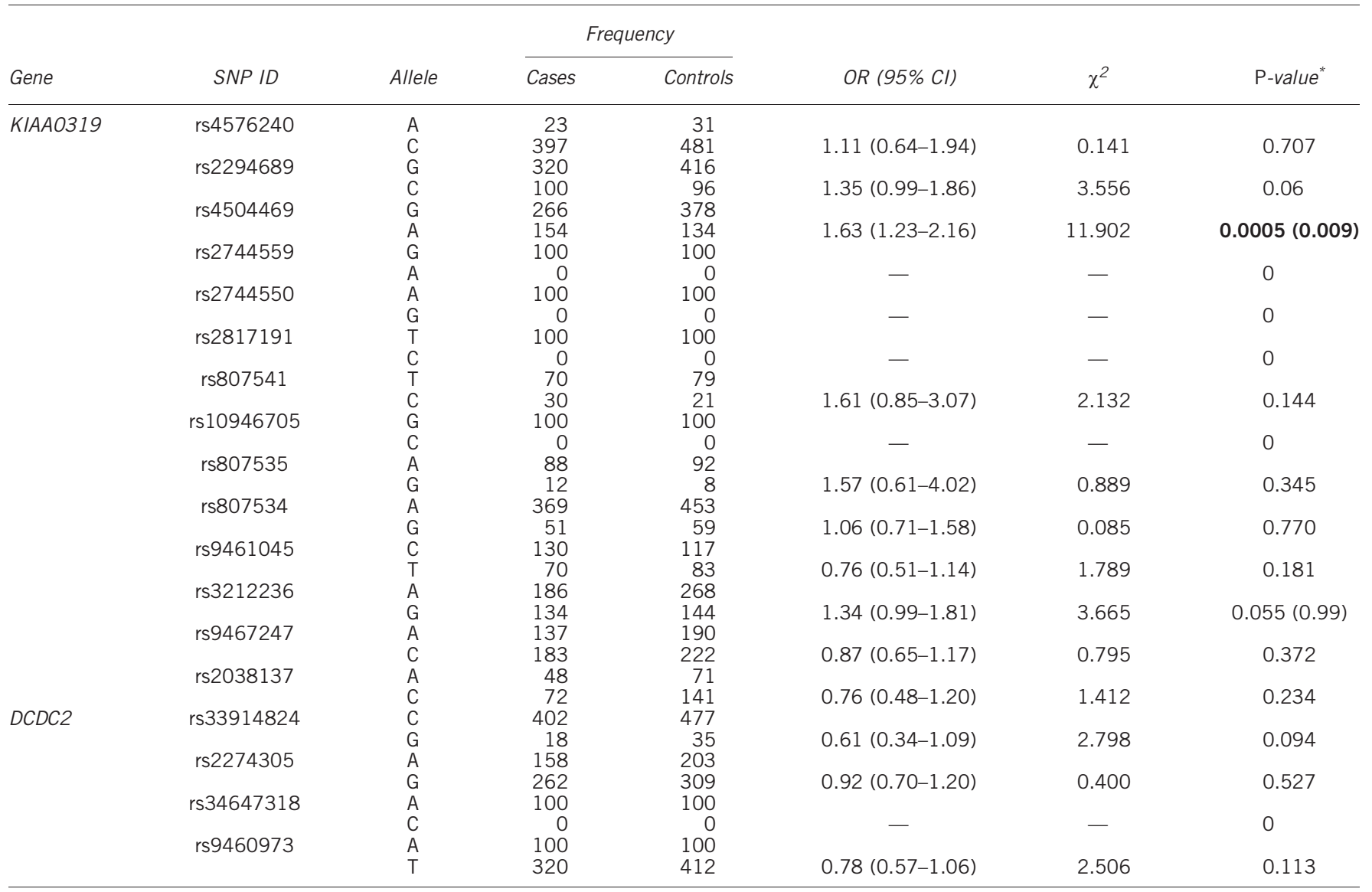

Abbreviations: $\mathrm{Cl}$, confidence interval; OR, odds ratio; SNP, single-nucleotide polymorphism. ${ }^{*} P$-values in the parenthesis were corrected by Bonferroni correction. Bold values indicate the significant $P$-value.

rs2294689, rs807541, rs9461045 and rs2038137 were observed in both cases and control groups without any significant association.

\section{DCDC2}

Statistical analysis of individual allele frequencies and homozygous genotype as well as genotypic models of four nonsynonymous exonic SNPs of DCDC2 failed to show any association.

\section{DISCUSSION}

KIAA0319 mainly expresses in different areas of human brain such as superior parietal cortex, primary visual cortex and occipital cortex, which are important for reading. ${ }^{10}$ It encodes for highly glycosylated membrane protein containing a large extracellular domain, a single transmembrane domain and a small intracellular C terminus. ${ }^{41,42}$ This protein also contains polycystic kidney disease repeats, which seem to be involved in the interaction between neurons and glial cells. ${ }^{42}$ RNA interference (RNAi) study has showed decrease in mRNA causing majority of the neurons to arrest in the proliferative ventricular area during embryonic development. Neuronal migration is a key step in the development of the neocortex, which provides the foundation for higher cognitive functions. ${ }^{23}$

Based on the earlier association studies and the role of KIAA0319 in neuronal migration, we selected both promoter and exonic SNPs of KIAA0319 and demonstrated the association of the exonic SNP of KIAA0319 rs4504469 with various phenotypes of DD. SNP rs4504469 is a nonsynonymous polymorphism leading to a substitution of amino acid alanine by threonine and its property changed from a neutral nonpolar residue to a neutral polar residue thereby, affecting the functioning of KIAA0319. Although our study did not analyze the haplotype, (rs4504469/rs2038137/rs2143340), which is associated with reduced expression of KIAA0319, one of the SNPs of the haplotype was identified as significant in our population. Meta-analysis of rs4504469 demonstrated global significant association of this SNP with DD. ${ }^{43}$ They suggest that direct effect of SNP rs4504469, that is, substitution of alanine by threonine could be modified by a second susceptibility allele and further effort is needed to clarify the underlying mechanism between rs4504469 variant and dyslexia risk. ${ }^{43}$

Genotype (GG-GA-AA) analyses of SNP rs4504469 was conducted for dominant model (GA + AA vs GG), recessive model (GG + GA vs AA) and additive model (GG vs GA vs AA). ${ }^{40}$ The trend toward significance of additive genetic model predicts that there is a linear gradient in risk between the GG, GA and AA genotypes. Significance of dominant model (odds ratio 1.76, 95\% confidence interval 1.22-2.55) showed the increased risk of DD with GA+AA genotype than the wild type, as wild-type genotype frequency was more in controls. Francks et al. ${ }^{11}$ found evidence for the association between rs4504469 with a broad range of reading-related cognitive abilities, such as phonological decoding, single-word reading and phonological awareness. In our study, we found association of the genotype AA and GA + AA of rs4504469 with reading and spelling measures, RAN speed, short-term memory and phoneme tasks. 
Table 3 Genotype distributions of SNPs of KIAAO319 and DCDC2 among children with developmental dyslexia and normal readers

\begin{tabular}{|c|c|c|c|c|c|c|c|c|c|}
\hline Gene & $S N P I D$ & Nucleotide change & \multicolumn{2}{|c|}{ Total no. of samples } & Genotype & Cases & Controls & OR $(95 \% \mathrm{Cl})$ & P-value* \\
\hline & & & & & $A C$ & 21 & 25 & $2.52(0.24-26.07)$ & 0.424 \\
\hline & & & & & $\mathrm{CC}$ & 188 & 228 & $2.47(0.26-23.98)$ & 0.419 \\
\hline & rs2294689 & $745 \mathrm{G}>C$ & 210 & 256 & GG & 160 & 208 & 1 (referent) & - \\
\hline & \multirow[t]{3}{*}{ rs4504469 } & \multirow[t]{3}{*}{$931 \mathrm{G}>\mathrm{A}$} & 210 & 256 & GG & 87 & 142 & 1 (referent) & - \\
\hline & & & & & GA & 92 & 94 & $1.60(1.08-2.36)$ & $0.018(0.324)$ \\
\hline & & & & & $A A$ & 31 & 20 & $2.53(1.36-4.71)$ & $0.002(0.036)$ \\
\hline & rs2744559 & $1698 \mathrm{G}>\mathrm{A}$ & 50 & 50 & GG & 50 & 50 & 1 (referent) & - \\
\hline & & & & & GA & 0 & 0 & - & 0 \\
\hline & & & & & $A A$ & 0 & 0 & - & 0 \\
\hline & & & & & TC & 0 & 0 & - & 0 \\
\hline & & & & & $\mathrm{CC}$ & 0 & 0 & - & 0 \\
\hline & rs807541 & $2694 \mathrm{~T}>\mathrm{C}$ & 50 & 50 & TT & 25 & 31 & 1 (referent) & - \\
\hline & & & & & TC & 20 & 17 & $1.46(0.63-3.36)$ & 0.374 \\
\hline & & & & & $\mathrm{CC}$ & 5 & 2 & $3.10(0.55-17.35)$ & 0.180 \\
\hline & rs10946705 & $2756 \mathrm{G}>C$ & 50 & 50 & GG & 50 & 50 & 1 (referent) & - \\
\hline & & & & & GC & 0 & 0 & - & 0 \\
\hline & & & & & CC & 0 & 0 & - & 0 \\
\hline & rs807535 & $2973 A>G$ & 50 & 50 & AA & 38 & 42 & 1 (referent) & - \\
\hline & & & & & $A G$ & 12 & 8 & $1.66(0.61-4.49)$ & 0.317 \\
\hline & & & & & GG & 0 & 0 & - & 0 \\
\hline & rs807534 & $3038 A>G$ & 210 & 256 & $A A$ & 173 & 213 & 1 (referent) & - \\
\hline & rs9467247 & $A>C$ & 160 & 206 & AA & 36 & 38 & 1 (referent) & - \\
\hline & & & & & $A C$ & 65 & 114 & $0.60(0.35-1.04)$ & 0.068 \\
\hline & & & & & CC & 59 & 54 & $1.15(0.64-2.07)$ & 0.633 \\
\hline & rs2038137 & $A>C$ & 60 & 106 & $A A$ & 10 & 13 & 1 (referent) & - \\
\hline & & & & & $A C$ & 28 & 45 & $0.81(0.31-2.09)$ & 0.661 \\
\hline & & & & & $\mathrm{CC}$ & 22 & 48 & $0.60(0.23-1.57)$ & 0.291 \\
\hline$D C D C 2$ & rs33914824 & $454 C>G$ & 210 & 256 & CC & 192 & 221 & 1 (referent) & - \\
\hline & & & & & CG & 18 & 35 & $0.59(0.32-1.08)$ & 0.084 \\
\hline & & & & & GG & 0 & 0 & - & 0 \\
\hline & rs2274305 & $661 A>G$ & 210 & 256 & $\mathrm{AA}$ & 38 & 45 & 1 (referent) & - \\
\hline & & & & & $A G$ & 82 & 113 & $0.86(0.51-1.44)$ & 0.565 \\
\hline & & & & & GG & 90 & 98 & $1.09(0.65-1.83)$ & 0.750 \\
\hline & rs34647318 & $1181 A>C$ & 50 & 50 & $A A$ & 50 & 50 & 1 (referent) & - \\
\hline & & & & & $A C$ & 0 & 0 & - & 0 \\
\hline & & & & & CC & 0 & 0 & - & 0 \\
\hline & rs9460973 & $1368 \mathrm{~A}>\mathrm{T}$ & 210 & 256 & $\mathrm{AA}$ & 50 & 50 & 1 (referent) & - \\
\hline & & & & & AT & 0 & 0 & - & 0 \\
\hline & & & & & TT & 160 & 206 & $0.78(0.50-1.21)$ & 0.262 \\
\hline
\end{tabular}

Abbreviations: $\mathrm{Cl}$, confidence interval; OR, odds ratio; SNP, single-nucleotide polymorphism.

${ }^{*} P$-values in the parenthesis were corrected by Bonferroni correction.

Bold values indicate the significant $P$-value. 
Table 4 Comparison of genotype frequencies of KIAAO319 and DCDC2 polymorphisms between children with developmental dyslexia and normal readers by dominant, recessive and additive models

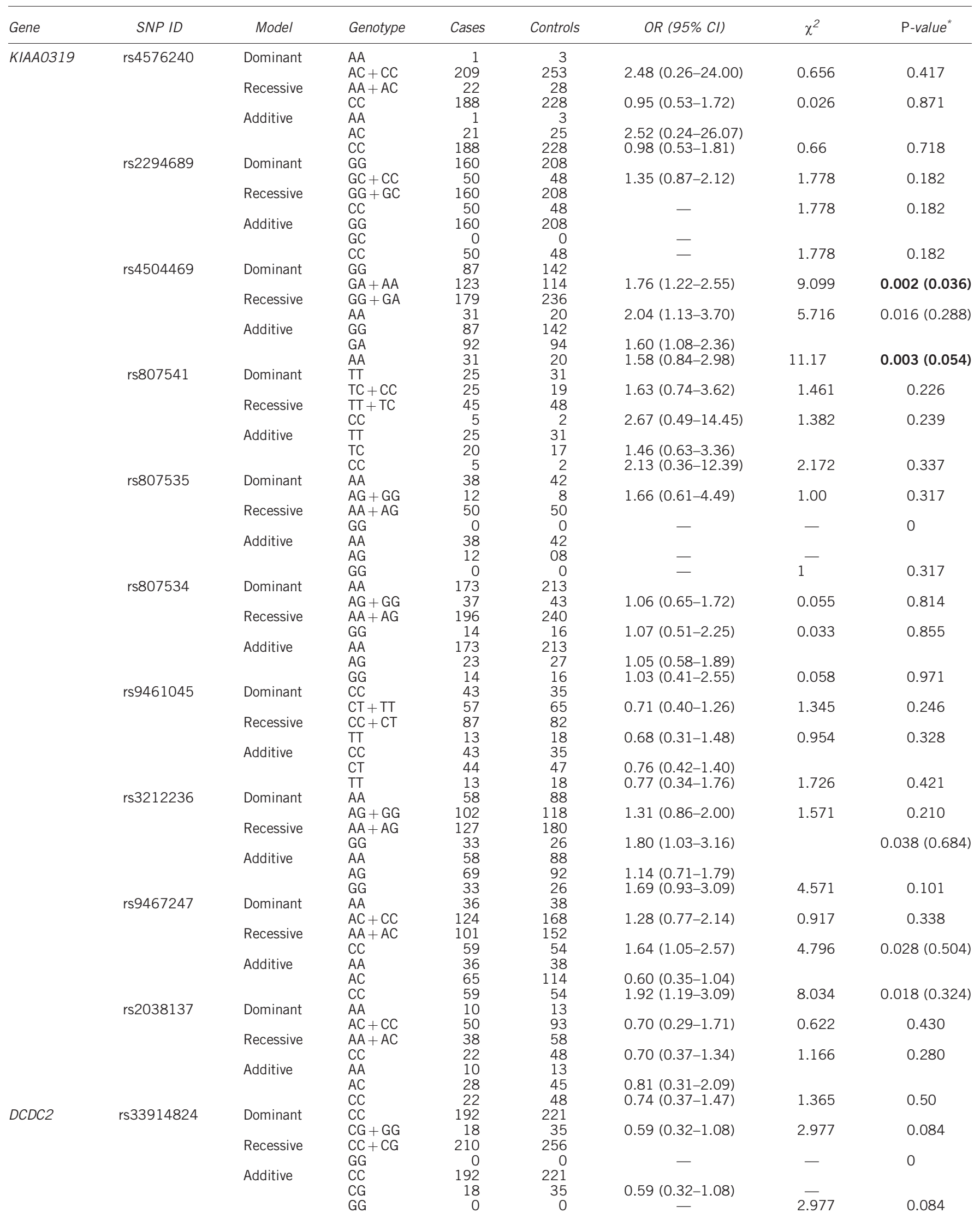


Table 4 (Continued)

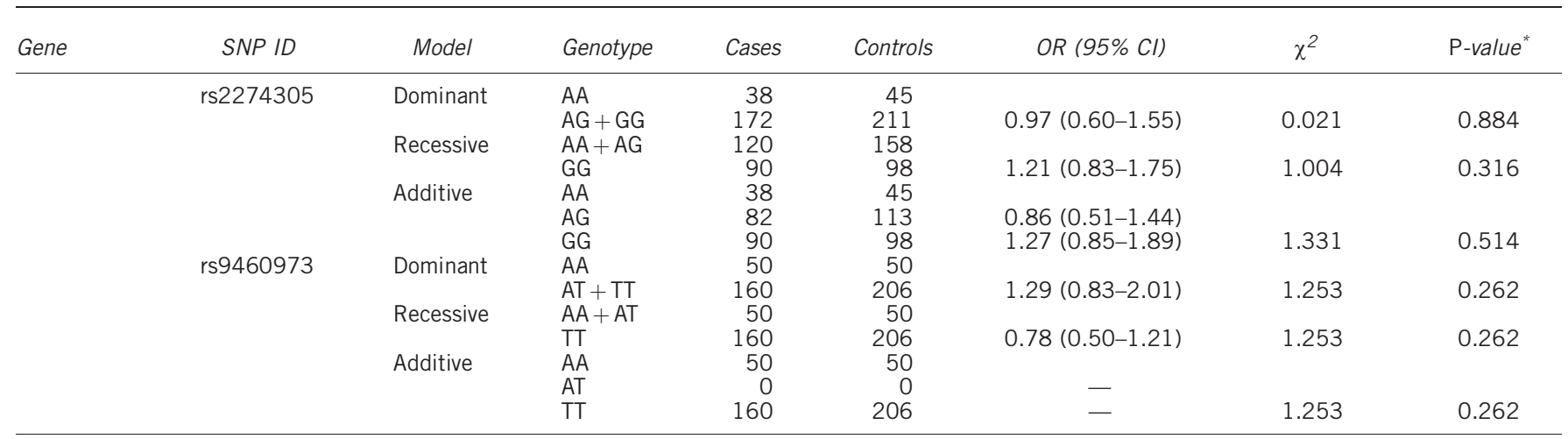

$\mathrm{Cl}$, confidence interval; OR, odds ratio; SNP, single-nucleotide polymorphism.

" $P$-values in the parenthesis were corrected by Bonferroni correction.

Bold values indicate the significant $P$-value.
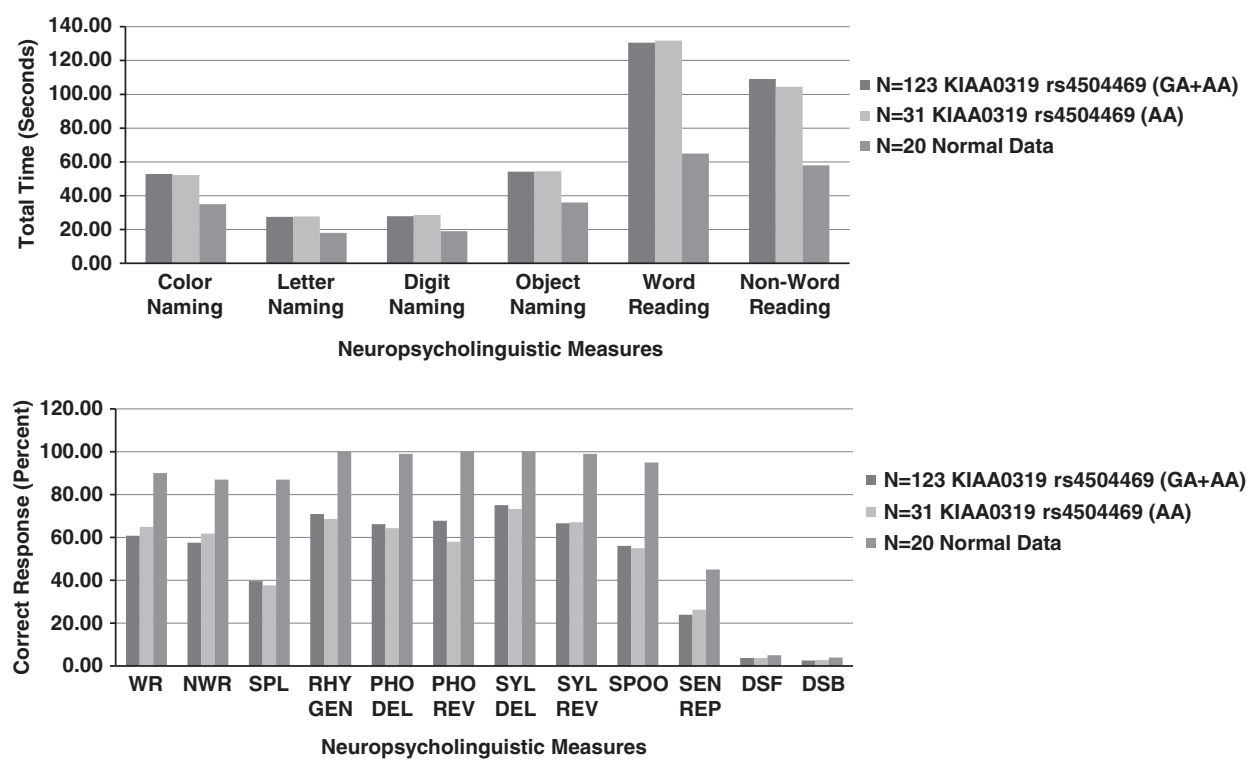

Figure 2 Performance on neuropsychological measures. WR, word reading; NWR, non-word reading; SPL, spelling; RHY GEN, rhyme generation; PHO DEL, phoneme deletion; PHO REV, phoneme reversal; SYL DEL, syllable deletion; SYL REV, syllable reversal; SPOO, spoonerism; SEN REP, sentence repetition; DSF, digit span (forward); DSB, digit span (backward).

Recently, luciferase construct in neuronal and non-neuronal cell lines demonstrated alter reporter gene expression by rs 9461045 and rs3212236 but rs9467247 did not show any alteration in the gene expression. ${ }^{24}$ The $\mathrm{G}$ allele of rs9461045 was shown to create a binding site for the transcriptional repressor OCT-1 but this SNP failed to show any association in our population, SNP rs3212236, which moderately affects the gene expression, was found as significant in genotype and allele frequency analysis as well as recessive model without testing for multiple corrections. ${ }^{24}$

Two candidate genes KIAA0319 and DCDC2 within the DYX2 locus have been reported to confer susceptibility to DD. Through a case-control study, we have provided evidence for the association of coding SNP rs4504469 of KIAA0319 with DD. To the best of our knowledge, this is the first genetic study that demonstrated KIAA0319 as a potential candidate gene for DD in Indian population. More studies with larger sample size will help reconfirming the findings reported here.

\section{ACKNOWLEDGEMENTS}

We thank the parents and students who participated in this study. We thank the funding agency Department of Science and Technology-Cognitive Science Initiative, Government of India, New Delhi (Grant no. SR/CSI/14/2008). We also thank Professor HA Ranganath for his help and encouragement; Avinash MV and Marita S for their help in sample collection, Surendranath Nishanimutt of Samveda Research and Training Institute, Davangere and Dr Gayathri Krishna of Brindavan Education Trust, Bangalore and Sita Krishnamurthy of Deepika Special School, Bangalore for helping in providing samples; Vimta Labs, Xcelris Labs and University of Mysore for providing facility to conduct this work. 
1 Katusic, S. K., Colligan, R. C., Barbaresi, W. J., Schaid, D. J. \& Jacobsen, S. J. Incidence of reading disability in a population-based birth cohort., 1976-1982., Rochester., Minn. Mayo. Clin. Proc. 76, 1081-1092 (2001).

2 Saviour, P., Padakannaya, P., Nishanimutt, S. \& Ramachandra, N. B. Familial patterns and biological markers of dyslexia. Ind. J. Hum. Gen 9, 21-29 (2009).

3 Williams, J. \& O'Donovan, M. C. The genetics of developmental dyslexia. Eur. J. Hum. Genet. 14, 681-689 (2006).

4 Kang, C. \& Drayna, D. Genetics of speech and language disorders. Annu. Rev. Genom. Hum. Genet. 12, 1-20 (2011).

5 Poelmans, G., Buitelaar, J. K., Pauls, D. L. \& Franke, B. A theoretical molecula network for dyslexia, Integrating available genetic findings. Mol. Psychiatry 16, 365382 (2011).

6 Fisher, S. E., Marlow, A. J., Lamb, J., Maestrini, E., Williams, D. F., Richardson, A. J. et al. A quantitative-trait locus on chromosome $6 p$ influences different aspects of developmental dyslexia. Am. J. Hum. Genet. 64, 146-156 (1999).

7 Grigorenko, E. L., Wood, F. B., Meyer, M. S. \& Pauls, D. L. Chromosome 6p influences on different dyslexia-related cognitive processes, further confirmation. Am. J. Hum. Genet. 66, 715-723 (2000)

8 Gayan, J. \& Olson, R. K. Genetic and environmental influences on orthographic and phonological skills in children with reading disabilities. Dev. Neuropsychol. 20, 483-507 (2001).

9 Kaplan, D. E., Gayán, J., Ahn, J., Won, T. W., Pauls, D., Olson, R. K. et al. Evidence for linkage and association with reading disability., on 6p21.3-22. Am. J. Hum. Genet. 70, 1287-1298 (2002)

10 Meng, H., Smith, S. D., Hager, K., Held, M., Liu, J., Olson, R. K. et al. DCDC2 is associated with reading disability and modulates neuronal development in the brain Proc. Natl Acad. Sci. USA 102, 17053-17058 (2005).

11 Francks, C., Paracchini, S., Smith, S. D., Richardson, A. J., Scerri, T. S., Cardon, L. R. et al. A 77- kilobase region of chromosome $6 \mathrm{p} 22.2$ is associated with dyslexia in families from the United Kingdom and from the United States. Am. J. Hum. Genet. 75, 1046-1058 (2004)

12 Cope, N., Harold, D., Hill, G., Moskvina, V., Stevenson, J., Holmans, P. et al. Strong evidence that KIAA0319 on chromosome $6 \mathrm{p}$ is a susceptibility gene for developmental dyslexia. Am. J. Hum. Genet. 76, 581-591 (2005).

13 Schumacher, J., Anthoni, H., Dahdouh, F., Konig, I. R., Hillmer, A. M., Kluck, N. et al. Strong genetic evidence of DCDC2 as a susceptibility gene for dyslexia. Am. J. Hum. Genet. 78, 52-62 (2006).

14 Rice, M. L., Smith, S. D. \& Gayan, J. Convergent genetic linkage and associations to language., speech and reading measures in families or probands with specific language impairment. J. Neurodevelop. Disord. 1, 264-282 (2009).

15 Wilcke, A., Weissfuss, J., Kirsten, H., Wolfram, G., Boltze, J. \& Ahnert, P. The role of gene DCDC2 in German dyslexics. Ann. Dyslexia 59, 1-11 (2009).

16 Lind, P. A., Luciano, M., Wright, M. J., Montgomery, G. W., Martin, N. G. \& Bates, T. C. Dyslexia and DCDC2, Normal variation in reading and spelling is associated with DCDC2 polymorphisms in an Australian population sample. Eur. J. Hum. Genet. 18, 668-673 (2010).

17 Elbert, A., Lovett, M. W., Cate-Carter, T., Pitch, A., Kerr, E. N. \& Barr, C. L. Genetic variation in the KIAA0319 5' region as a possible contributor to dyslexia. Behav. Genet. 41, 77-89 (2011).

18 Newbury, D. F., Paracchini, S., Scerri, T. S., Winchester, L., Addis, L., Richardson, A. J. et al. Investigation of dyslexia and SLI risk variants in reading- and language-impaired subjects. Behav. Genet. 41, 90-104 (2011).

19 Scerri, T. S., Morris, A. P., Buckingham, L., Newbury, D. F., Miller, L. L., Monaco, A. P. et al. DCDC2, KIAAO319 and CMIP are associated with reading-related traits. Biol. Psychiatry 70, 237-245 (2011).

20 Harold, D., Paracchini, S., Scerri, T., Dennis, M., Cope, N., Hill, G. et al. Further evidence that the KIAA0319 gene confers susceptibility to developmental dyslexia. Mol. Psychiatry 11, 1085-1191 (2006).

21 Paracchini, S., Thomas, A., Castro, S., Lai, C., Paramasivam, M., Wang, Y. et al. The chromosome $6 \mathrm{p} 22$ haplotype associated with dyslexia reduces the expression of KIAA0319, a novel gene involved in neuronal migration. Hum. Mol. Genet. 15, 1659-1666 (2006).
22 Dennis, M. Y., Paracchini, S., Scerri, T. S., Prokunina-Olsson, L., Knight, J. C., WadeMartins, R. et al. A common variant associated with dyslexia reduces expression of the KIAA0319 gene. PLoS Genet. 5, e1000436 (2009).

23 Couto, J. M., Livne-Bar, I., Huang, K., Xu, Z., Cate-Carter, T., Feng, Y. et al. Association of reading disabilities with regions marked by acetylated $\mathrm{H} 3$ histones in KIAA0319. Am. J. Med. Genet. B Neuropsychiatr. Genet. 153B, 447-462 (2010).

24 Luciano, M., Lind, P. A., Duffy, D. L., Castles, A., Wright, M. J., Montgomery, G. W. et al. A haplotype spanning KIAA0319 and TTRAP is associated with normal variation in reading and spelling ability. Biol. Psychiatry 62, 811-817 (2007).

25 Paracchini, S., Steer, C. D., Buckingham, L. L., Morris, A. P., Ring, S., Scerri, T. et al Association of the KIAA0319 dyslexia susceptibility gene with reading skills in the general population. Am. J. Psychiatry 165, 1576-1584 (2008).

26 Deffenbacher, K. E., Kenyon, J. B., Hoover, D. M., Olson, R. K., Pennington, B. F., DeFries, J. C. et al. Refinement of the $6 \mathrm{p} 21.3$ quantitative trait locus influencing dyslexia, linkage and association analyses. Hum. Genet. 115, 128-138 (2004).

27 Czamara, D., Bruder, J., Becker, J., Bartling, J., Hoffmann, P., Ludwig, K. U. et al. Association of a rare variant with mismatch negativity in a region between KIAA0319 and DCDC2 in dyslexia. Behav. Genet. 41, 110-119 (2011).

28 Marino, C., Meng, H., Mascheretti, S., Rusconi, M., Cope, N., Giorda, R. et al. DCDC2 genetic variants and susceptibility to developmental dyslexia. Psychiatr. Genet. 22 25-30 (2012).

29 Ludwig, K. U., Roeske, D., Schumacher, J., Schulte-Körne, G., König, I. R., Warnke, A. et al. Investigation of interaction between DCDC2 and KIAA0319 in a large German dyslexia sample. J. Neural. Transm. 115, 1587-1589 (2008).

30 Brkanac, Z., Chapman, N. H., Matsushita, M. M., Chun, L., Nielsen, K., Cochrane, E. et al. Evaluation of candidate genes for DYX1 and DYX2 in families with dyslexia. Am. J. Med. Genet. B 144B, 556-560 (2007).

31 Peng-xiang, Z., Han-rong, W., Zeng-chun, L., Xu-dong, C., Li-juan, P., Lan, Y. et al. Association of polymorphisms in the DCDC2 gene with developmental dyslexia in the Han Chinese. Chin. Med. J. 125, 622-625 (2012).

32 Zhong, R., Yang, B., Tang, H., Zou, L., Song, R., Zhu, L. Q. et al. Meta-analysis of the association between DCDC2 polymorphisms and risk of dyslexia. Mol. Neurobiol. 47, 435-442 (2013)

33 Paracchini, S., Ang, Q. W., Stanley, F. J., Monaco, A. P., Pennell, C. E., Whitehouse, A J. et al. Analysis of dyslexia candidate genes in the Raine cohort representing the general Australian population. Genes. Brain Behav. 10, 158-165 (2011).

34 Venkatesh, S. K., Siddaiah, A., Padakannaya, P. \& Ramachandra, N. B. An examination of candidate gene SNPs for dyslexia in an Indian Sample. Behav. Genet. 41, 105-109 (2011)

35 Raven, J. C. Mental tests used in genetic studies, The performance of related individuals on tests mainly educative and mainly reproductive MSc thesisUniv London, 1936).

36 Joshi, R. M. \& Aaron, P. G. in Literacy Acquisition Assessment and Instruction: The Role of Phonology, Orthography and Morphology (eds Joshi, R. M., Kaczmanek, B. \& Leong, C. K.) 153-161 (IOS Press, Amsterdam/Holland, 2003).

37 Oldfield, R. C. The assessment and analysis of handedness, the Edinburgh inventory. Neuropsychologia 9, 97-113 (1971).

38 Das, J. P. \& Naglieri, J. A. Cognitive Assessment System, Interpretive Handbook Riverside Publishing, Itasca, IL, 1997).

39 Pershad, D. \& Wig, N. N. A battery of simple tests of memory for use in India. Neurol. India 24, 86-93 (1976).

40 Salanti, G., Southam, L., Altshuler, D., Ardlie, K., Barroso, I., Boehnke, M. et al. Underlying genetic models of inheritance in established type 2 diabetes associations. Am. J. Epidemiol. 170, 537-545 (2009).

41 Galaburda, A. M., LoTurco, J., Ramus, F., Fitch, R. H. \& Rosen, G. D. From genes to behavior in developmental dyslexia. Nat. Neurosci. 9, 1213-1217 (2006).

42 Velayos-Baeza, A., Toma, C., Paracchini, S. \& Monaco, A. P. The dyslexia-associated gene KIAA0319 encodes highly $\mathrm{N}$ - and $\mathrm{O}$-glycosylated plasma membrane and secreted isoforms. Hum. Mol. Genet. 17, 859-871 (2008).

43 Zou, L., Chen, W., Shao, S., Sun, Z., Zhong, R., Shi, J. et al. Genetic Variant in KIAA0319, But Not in DYX1C1., is associated with risk of dyslexia, an integrated meta-analysis. Am. J. Med. Genet. B Neuropsychiatr. Genet. 159B, 970-976 (2012)

Supplementary Information accompanies the paper on Journal of Human Genetics website (http://www.nature.com/jhg) 